\title{
Erratum to: Prognostic relevance of microsatellite instability in pT3NOMO colon cancer: a population-based study
}

\author{
Francesco Iachetta ${ }^{1}$. Federica Domati ${ }^{1,4}$. Luca Reggiani-Bonetti ${ }^{1}$. \\ Valeria Barresi ${ }^{2}$ - Giulia Magnani ${ }^{1}$ - Luigi Marcheselli ${ }^{1}$ - Claudia Cirilli ${ }^{3}$. \\ Monica Pedroni ${ }^{1}$
}

Published online: 26 October 2015

(C) SIMI 2015

\section{Erratum to: Intern Emerg Med DOI 10.1007/s11739-015-1285-6}

Authors would like to correct the incorrect data reported in the abstract and in the text about the adjuvant chemotherapy and the data relative to correlation with MSI and Mucinous histology.

The complete abstract text should read as below:

\begin{abstract}
Although surgery alone represents a curative approach for patients with pT3N0M0 colon cancer, about 15-20\% of these patients develop a relapse of disease. Microsatellite instability (MSI) is one of the most important molecular markers in colorectal cancer. The aim of this study was to investigate the prognostic relevance of MSI in all pT3N0M0 tumors recorded in the Cancer Registry of the Province of Modena-(Northern Italy) within the 2002-2006 period in patients who showed a relapse of disease during the 5-year period of follow-up (59 cases). They were compared to 59 controls similar in clinical and
\end{abstract}

pathological features but with good prognosis. 32 patients of the 118 cases received adjuvant chemotherapy, 16 $(27 \%)$ in the group of relapses and $16(27 \%)$ in the controls group. MSI status was tested using BAT25, BAT26, NR24, and CAT25 fluorescent-labeled mononucleotide markers. The overall prevalence of MSI was $12.7 \%$ (15 of 118 cases). MSI was detected mainly in mucinous adenocarcinoma ( $p<0.003)$, in high-grade tumors $(p<0.008)$, in right-sided neoplasms $(p=0.05)$, and in patients with a better prognosis, though the difference was not statistically significant (11/59 patients $-18.6 \%$ vs $4 / 59$ patients $-6.7 \%$; OR 0.36, CI $95 \% 0.11-1.15$; $p=0.08$ ). However, in multivariate analysis, MSI status becomes the strongest independent factor associated with relapse (OR 0.21 , CI $95 \% 0.06-0.82 ; p=0.024)$, together with mucinous histological type (OR 6.08, CI $95 \%$ 1.16-31.8; $p=0.032$ ). MSI is a relevant prognostic factor in stage pT3N0M0 colon cancer suitable to discriminate those patients with a high risk of relapse.

The online version of the original article can be found under doi:10.1007/s11739-015-1285-6.

Federica Domati

federica.domati@unimore.it

1 Department of Diagnostic, Clinical and Public Health Medicine, University of Modena and Reggio Emilia, Modena, Italy

2 Department of Pathology, University of Messina, Messina, Italy

3 Modena Cancer Registry, Modena, Italy

4 Department of Internal Medicine, Medicina I, University of Modena and Reggio Emilia, Via del Pozzo 71, 41100 Modena, Italy 